\title{
Complejidad argumentativa individual escrita en estudiantes universitarios ingresantes $y$ avanzados
}

\section{Written individual argumentative complexity of first-year and senior university students}

\author{
María Agustina Tuzinkievicz ${ }^{\mathrm{a}}$, Nadia Soledad Peralta ${ }^{\mathrm{b}, *}$, Mariano Castellaro ${ }^{\mathrm{b}}$, Cristián Santibáñez

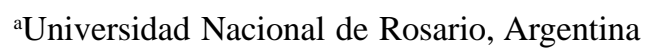 \\ ${ }^{\mathrm{b}}$ Instituto Rosario de Investigaciones en Ciencias de la Educación; Universidad Nacional de Rosario, Argentina \\ 'Universidad Católica de la Santísima de Concepción, Chile
}

\section{Resumen}

La investigación compara la complejidad argumentativa individual escrita en tareas sociocientíficas de estudiantes universitarios de Psicología en función del momento de cursado (ingresante/avanzado) y de la presencia de un sistema de representación externa adicional (gráfico). Participaron 72 estudiantes de una universidad argentina. Estos emitieron opinión escrita sobre una situación sociocientífica con argumentos a favor y en contra, y un grupo dispuso de un sistema de información adicional (gráfico). Se codificaron las producciones según: (a) si se trataba de un texto argumentativo; (b) en los textos argumentativos, se consideraron cantidad de argumentos, diferencia de opinión principal, estructura argumentativa, sesgo confirmatorio y (c) uso de información. Los resultados indicaron una relación entre los estudiantes ingresantes y la producción de textos no argumentativos, por un lado, y de los estudiantes avanzados y la producción de textos argumentativos, por el otro. Se discuten las implicancias de los resultados en relación a la comprensión teórica de la competencia argumentativa y la creación de material educativo para la formación universitaria.

Palabras clave: argumentación, educación superior, gráficos, escritura.

Para citar este artículo:

Tuzinkievicz, M. A., Peralta, N. S., Castellaro, M., \& Santibañez, C. (2018). Complejidad argumentativa individual escrita en estudiantes universitarios ingresantes y avanzados. Liberabit, 24(2), 231-247. https://doi.org/10.24265/liberabit.2018.v24n2.05

\begin{abstract}
The research compares the written individual argumentative complexity in socio-scientific tasks of psychology university students, based on the year of study (first-year/senior student) and the presence of an additional external representation system (graph). Seventy-two (72) students from an Argentine university participated in the study. They gave their written opinion about a socio-scientific situation that included arguments for and against it, and a group received an additional information system (graph). The productions were coded according to: (a) whether it was an argumentative text; (b) the number of arguments, difference from the main opinion, argumentative structure, and confirmatory bias in the argumentative texts; and (c) the use of information. The results showed a relationship between first-year students and the production of non-argumentative texts, and a relationship between senior students and the production of argumentative texts. The results of the theoretical conceptualization of the argumentative competence, and the design of educational materials for university training are discussed.
\end{abstract}

Keywords: argumentation, higher education, graphs, writing.

Este es un artículo Open Access bajo la licencia Creative Commons Atribución-NoComercial-CompartirIgual 4.0

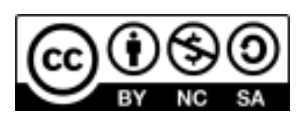




\section{Introducción}

Existe una gran diversidad de maneras de abordar la investigación sobre argumentación y esta variación se manifiesta tanto en las preguntas que guían las investigaciones como en las perspectivas de análisis, métodos y unidades de análisis adoptados (Leitão, 2000).

Sin embargo, vale resaltar que la literatura sobre la argumentación acuerda en, al menos, tres puntos fundamentales. Primero, el carácter normativo de la práctica argumentativa (Bermejo-Luque, 2011; Eemeren \& Grootendorst, 2004; Johnson, 2000; Siegel \& Biro, 2008; Tindale, 2015), lo cual deriva del hecho de que los agentes involucrados en un intercambio argumentativo presuponen algún estándar a partir del cual producen argumentos y evalúan a los mismos. En segundo lugar, también hay consenso en el carácter social del fenómeno (Hample \& Irions, 2015; Jacobs, 2016; Santibáñez, 2014, 2016), ya que el término argumentación es utilizado para referirse a la actividad de hacer afirmaciones, desafiarlas, darles apoyo mediante la producción de razones y refutar críticas (Popa, 2016) entre agentes que concurren en torno a la clarificación de una duda o en torno al convencimiento. En tercer lugar, se acuerda también en su carácter dialógico (García-Milá et al., 2015; Leitão, 2000; Smokrovic, 2015), vale decir, se observa que siempre una duda u oposición implica una audiencia a la que está dirigida, tanto en una comunicación oral como en una escrita, incluso en aquellos casos en los que un mismo agente evalúa información en un proceso de diálogo interno para utilizarla o no como razones para apoyar un punto de vista. Desde una perspectiva analítica, y como corolario de estos puntos de acuerdo, para argumentar se hace necesario diferenciar las afirmaciones de sus justificaciones, como también las afirmaciones propias de las ajenas y la relación entre ellas a favor o en contra de un punto de vista (García-Milá et al. 2015).

Que la actividad argumentativa suponga estos tres elementos básicos (normatividad, sociabilidad y dialogicidad), significa que los agentes naturales exhibirán distintos grados de complejidad argumentativa de acuerdo con los tipos de controversias y opiniones a las que se enfrentan, el contexto o lugar social en el que argumentan, y el tipo de rol o función que ocupan en los diálogos. Para comenzar a rastrear de forma algo más sistemática el problema de la complejidad argumentativa, este estudio se propuso analizar la argumentación escrita en estudiantes universitarios. De forma específica, nos parece de gran utilidad para el conocimiento y diagnóstico de la situación académica de estudiantes de Psicología y, de manera ampliada, de otras carreras de grado afines. Comparar las producciones de estudiantes avanzados e iniciales permitiría indagar sobre el efecto general que puede llegar a tener la formación universitaria sobre su capacidad argumentativa y crítica.

En este contexto, el objetivo general de este trabajo de investigación fue comparar la complejidad argumentativa individual escrita en tareas sociocientíficas en estudiantes universitarios de la carrera de Psicología en función del momento de cursado (ingresantes o avanzados) y de la presencia (o no) de un sistema de representación externa adicional (gráfico de frecuencia) al texto fuente sobre el cual se argumenta. Se plantearon los siguientes objetivos específicos:

a. Determinar si los estudiantes participantes realizan producciones escritas argumentativas.

b. Analizar la complejidad argumentativa escrita de las producciones según el número de argumentos, estructura argumentativa, diferencia de opinión principal y el sesgo de información.

c. Indagar si existen diferencias en relación al número de argumentos, estructura argumentativa, diferencia de opinión principal y el sesgo de información entre estudiantes ingresantes y avanzados.

d. Analizar la influencia de un sistema de información adicional (gráfico de frecuencia) al texto base sobre los indicadores de complejidad argumentativa escrita mencionados en el punto anterior. 
e. Identificar si hay diferencias entre ambos grupos de estudiantes respecto del uso de información adicional (gráficos).

Las tareas sociocientíficas (CSC) consisten en eventos, hechos o problemáticas relacionados con la tecnología y la ciencia, caracterizados por ser controvertidos, actuales y de interés social. Presentan un amplio espectro de implicaciones y perspectivas para su resolución (Carrión \& Castro, 2012) y no resultan del todo ajenas a ningún individuo (España \& Prieto, 2010).

Se las considera también útiles, ya que contribuyen a desestabilizar la visión común de la ciencia; y neutra y objetiva, para romper con la idea de que para estos problemas hay una solución única y absoluta, promoviendo así el pensamiento complejo y la variedad de perspectivas. Por este motivo, se considera que ofrecen un contexto apropiado para la formación de estudiantes universitarios (González \& Puig, 2017) y en su resolución se pone en juego la competencia argumentativa (España \& Prieto, 2010).

A su vez, se considera en este trabajo a los gráficos y tablas como representaciones externas. Estos tienen un valor social y cultural para el análisis y uso epistémico de la información (García-Milá et al., 2015).

Se denomina «crítica gráfica» a la habilidad de utilizar información brindada en gráficos para confirmar o refutar teorías tanto ajenas como propias y para darle sentido a los datos que se presentan (Roth, Pozzer-Ardenghi, \& Han, 2005).

Numerosos trabajos al respecto han demostrado que el uso epistémico de estas representaciones depende del conocimiento académico, el conocimiento específico del contenido representado y, finalmente, de los sistemas de representación y el contexto y objetivos de la tarea (García-Milá et al., 2015).

\section{Definiendo la noción de complejidad argumentativa}

Una de las teorías en el campo especializado del estudio de la argumentación que parte de estos tres puntos teóricos de convergencia es la pragma-dialéctica (Eemeren, 2018). Para la teoría pragma-dialéctica, la argumentación es una actividad verbal, social y racional que busca convencer a un crítico razonable acerca de la aceptabilidad de un punto de vista, presentando una constelación de una o más proposiciones para justificarlo. La funcionalidad de la argumentación está entonces dirigida a la resolución crítica de una diferencia de opinión a través de medios verbales. En consecuencia, se observa la argumentación como una actividad en la que hay una discusión explícita o implícita entre dos sujetos que tienen distintas posiciones respecto de una proposición.

Aunque no utilicen el término de forma directa, la teoría pragma-dialéctica comienza el tratamiento y estudio de la complejidad argumentativa en el mismo punto donde comienza la argumentación en la práctica: en la diferencia de opinión (Eemeren, Grootendorst \& Snoeck-Henkemans, 2002). Para los autores, la diferencia de opinión puede ser explícita o implícita. Especialmente en los textos escritos, ya que es una sola parte la que se expresa, la diferencia de opinión tiende a estar implícita. Sin embargo, el punto de vista del opositor, ya sea real o imaginario, siempre se supone.

No obstante, estén o no presentes, esta teoría parte de la base de que la argumentación siempre tiene el objetivo de convencer a críticos potenciales. Por este motivo, un análisis de producciones escritas debería comenzar con la distinción del tipo de diferencia de opinión que estos presentan. La diferencia de opinión única no mixta es la forma más simple en la que un punto de vista se encuentra con una duda del lado del interlocutor. Esto se manifiesta en la argumentación escrita, donde no hay interlocutor presente, cuando el protagonista expresa su punto de vista sin hacer referencia al del antagonista. La pragma-dialéctica justifica este hecho diciendo que ante la duda del 
adversario, real o supuesta, el protagonista no siente el compromiso de defender su opinión.

En el caso de la diferencia de opinión múltiple no mixta, se mantiene la suposición de la duda del otro, pero el punto de vista expresado se relaciona con más de una proposición. La diferencia de opinión única mixta consiste en que, respecto de una misma proposición, se adoptan dos puntos de vista distintos; es decir, un lado adopta una postura respecto al tema en discusión y el otro responde adoptando la contraria. En el plano de la argumentación escrita, esto consistiría en la inclusión de un argumento contrario en la propia argumentación. Finalmente, la diferencia de opinión múltiple mixta implica una producción que contenga varios argumentos propios y contrarios.

Otra dimensión para estudiar la complejidad argumentativa desde el punto de vista de la pragmadialéctica se relaciona con la importancia de evaluar la estructura argumentativa. Esta puede consistir en una argumentación simple que se caracteriza por la presencia de un argumento único o argumentación compleja, caracterizada por la presencia de más de un argumento. A su vez, según el nexo entre los argumentos, puede dividirse en las siguientes categorías: múltiple, la defensa del punto de vista consiste en múltiples argumentos alternativos presentados uno luego del otro, de manera independiente y teniendo todos el mismo peso; coordinada, aquí los argumentos no son defensas alternativas a la posición tomada sino que constituyen un único intento de defensa de la posición, consistente en la combinación de argumentos que por separado no tienen suficiente fuerza defensiva; y subordinada, en este tipo de argumentación, quien argumenta anticipa que algunas partes requerirán más defensa. Ese argumento será un subpunto de vista que deberá ser defendido por una subargumentación. Esta subargumentación puede resultar también en su totalidad o tener componentes que resulten subsubpuntos de vista que requerirán una subsubargumentación y así sucesivamente. Se constituye así una cadena de razonamiento. A las categorías de análisis detalladas anteriormente, se le suma la de número de argumentos como un indicador más para dar cuenta de la complejidad argumentativa escrita.

Por otro lado, también se analiza como indicador el uso o no de la información brindada en la tarea propuesta. García-Milá et al. (2015) realizaron un estudio con la finalidad de evaluar cómo los estudiantes españoles de nivel secundario y universitario utilizan informaciones que apoyan o refutan la propia tesis. Dicha información fue brindada en forma de gráficos, para lo cual se utilizó el concepto de sesgo confirmatorio. Para el análisis del uso de la información brindada en gráficos por parte de los sujetos se utilizaron las siguientes categorías: argumento dado (información aportada por los gráficos), argumento seminuevo (aquella información relacionada con la mostrada en los gráficos, pero modificada mediante la adición de algún dato o consideración) y argumento nuevo (utilización de información pertinente que el participante añade a su argumentación). Para evaluar el tipo de tratamiento que se le da a la información contraria a la posición propia que es brindada en los gráficos, utilizaron los siguientes criterios de análisis: se ignora (no se hace mención a la información adicional), se lee incorrectamente (utilizan parte o toda la información, pero erróneamente), se lee parcialmente (no se ignoró toda la información contraria a la tesis, pero sí una parte de ella) y se lee subjetivamente (la lectura incorrecta de la información se da por un tinte afectivo o relativo a las experiencias de la persona). Los resultados de este estudio mostraron que la comprensión de los gráficos es buena y no existen diferencias respecto del nivel educativo. También se observó, de manera general, una buena competencia argumentativa, en tanto que los participantes fueron capaces de integrar aspectos a favor y en contra en sus textos y utilizaron las informaciones dadas adicionalmente, así como también aportaron nueva información. 


\section{El problema de la calidad argumentativa escrita}

Investigaciones recientes han estudiado la calidad de las producciones argumentativas escritas en estudiantes universitarios. Más allá de la diversidad de enfoques, todas coinciden en el bajo desempeño argumentativo de los estudiantes, incluso partiendo de diferentes enfoques conceptuales de base. Este dato, en cierta medida preocupante, marca la relevancia de estudiar la argumentación en este nivel educativo. A continuación, se describen algunos antecedentes que han concluido en el sentido anterior.

Bajo el nombre de complejidad discursiva, Hammer y Noemí (2016) estudiaron escritos de universitarios chilenos para establecer una relación entre la misma y el pensamiento crítico, entendiendo por este último, una acción que pone en tela de juicio el conocimiento disponible expresado en forma de discurso, por lo tanto, analizado desde el análisis del contenido. Analizaron la complejidad discursiva como la suma total de los tipos de relaciones que los sujetos producen en sus discursos. Los resultados indicaron que la mayoría de los participantes realizaron producciones escritas con baja complejidad discursiva. Sin embargo, se observó una concordancia entre sujetos que mostraron alta o muy alta complejidad discursiva y alto o muy alto pensamiento crítico.

Por otro lado, López y Padilla (2011) estudiaron los grados de complejidad argumentativa de escritos de estudiantes universitarios ingresantes en Argentina con el objeto de valuar la productividad de los instrumentos pragma-dialécticos con miras a evaluar el grado de competencia argumentativa de los estudiantes al inicio de los estudios universitarios. Los resultados mostraron que casi la mitad de la muestra realizó producciones sin argumentos y, atendiendo a los tipos de diferencias de opinión, únicamente se observaron la opción única mixta y la única no mixta.

Arrosi, Axelrud, D’Agostino y Eisner (2003), en un trabajo con estudiantes ingresantes en Argentina, analizaron las producciones escritas que fueron elaboradas a partir de una consigna y los resultados mostraron que los jóvenes eran capaces de plantear un punto de vista y fundamentarlo. Sin embargo, las producciones no incluían un buen nivel de polifonía, es decir, no realizaron la introducción de otras opiniones diferentes a las suyas o las introdujeron, pero no fueron aprovechadas para la justificación de su punto de vista. Resultados similares se encuentran en los estudios de Errázuriz y Fuentes (2010).

Estos resultados coinciden con los planteados por Carrillo (2010), los cuales ponen de manifiesto las dificultades que los estudiantes experimentan en la producción de textos, puesto que se observó un porcentaje relevante de textos que carecen de argumentos.

\section{Método}

Se utilizó un diseño cuasiexperimental compuesto por dos variables independientes: a) momento de cursado de la carrera (estudiante ingresante o estudiante avanzado) y b) presencia de un sistema de representación externa adicional (gráfico de frecuencia) al texto fuente utilizado como base de la argumentación escrita (presencia o ausencia de dicho sistema). Aunque, en sentido estricto, la variable momento de cursado no es manipulable per se, fue considerada como independiente en el contexto del presente estudio. La variable dependiente principal fue el grado de complejidad argumentativa escrita, analizada a partir de diferentes indicadores específicos (ver sección Análisis de datos).

Por tanto, el diseño permitió comparar la complejidad argumentativa entre cuatro condiciones, a partir de la combinación entre dos momentos de cursado (avanzados e ingresantes) y la presencia o ausencia de un sistema informacional (gráfico adicional). Si bien la asignación de los participantes a cada condición se basó en un criterio aleatorio, el diseño fue considerado cuasiexperimental por las restricciones que plantea el propio contexto universitario en el que habitualmente se desarrolla este tipo de estudio. 


\section{Participantes}

Se constituyó una muestra no probabilística por disponibilidad. Los participantes fueron contactados en clases de la Facultad de Psicología de la Universidad Nacional de Rosario (UNR) con previo permiso de los docentes a cargo. Inicialmente, se preveía contar con 80 estudiantes de psicología: 40 avanzados y 40 ingresantes. Finalmente, la muestra quedó conformada por 72 sujetos: 41 estudiantes avanzados (que cursaban los últimos 2 años de la carrera) y 31 ingresantes. Todos los sujetos fueron en su mayoría mujeres y con un promedio de edad de $23.82(\mathrm{D}=5.72$ ). A su vez, cada subgrupo fue dividido en dos condiciones: 21 estudiantes avanzados realizaron la actividad sin información adicional (gráfico de frecuencia complementario a un texto base) y los 20 restantes la realizaron con dicha información adicional; mientras que 18 estudiantes ingresantes realizaron la actividad sin información adicional y 13 contando con la misma.

\section{Instrumentos}

En primer lugar, se elaboró una actividad previa al estudio propiamente dicho que solo se aplicó al subgrupo de estudiantes (avanzados o ingresantes) que realizarían la actividad con información adicional (gráfico de frecuencias) al texto fuente. El propósito de esta actividad fue asegurar que dichos estudiantes contaban con una capacidad básica de comprensión del sistema representacional. Si un sujeto no registraba este requisito, no era incluido en la condición respectiva. La actividad consistió en 4 gráficos sobre diferentes temáticas (esperanza de vida según región, uso de navegadores para ingresar a Facebook, tasa de embarazo y producción transgénica), cada uno de ellos con 3 afirmaciones sobre los datos del gráfico de las cuales los participantes debían seleccionar la correcta y luego fundamentar brevemente por escrito la elección.

En segundo lugar, el texto que se brindó a todos los estudiantes era un escrito construido ad hoc que incluía una breve descripción de un asunto sociocientífico y argumentos a favor y en contra de tal situación, presentados de una manera neutral. Se consideraban asuntos o cuestiones sociocientíficas (CSC) a los eventos, hechos o problemáticas relacionados con la tecnología y la ciencia, que se caracterizan por ser controvertidos, actuales y de interés social, y presentan un amplio espectro de implicaciones y perspectivas para su resolución (Carrión \& Castro, 2012). El problema sociocientífico planteado fue la apertura o cierre de una central nuclear, donde se le brindaba a los sujetos argumentos a favor y en contra del cierre de la misma. Luego se les solicitaba a los participantes que dieran su opinión por escrito de la siguiente manera: «Ante el hecho del posible cierre permanente de la Central Nuclear de Garoña, el Ministerio de Industria creará una comisión conformada por ciudadanos expertos y no expertos para evaluar la situación. Usted es un ciudadano vasco y le interesa participar de dicho comité, por lo cual debe redactar un texto dando a saber su posición respecto del cierre o no de la Central».

Por último, a los participantes que trabajaron con información adicional se les brindó, junto al texto fuente, información extra respecto de la temática en forma de gráfico. Se les presentaron a los sujetos 4 gráficos, dos de ellos con información que podía ser usada como argumento a favor del cierre de la central y dos con información en contra del cierre de la central. Estos gráficos fueron extraídos del trabajo de García-Milá et al. (2015).

\section{Procedimiento}

Se convocó a los estudiantes para realizar la actividad en un aula de la Facultad durante 45 minutos fuera del horario de clases. Se dejó expresa constancia de la participación voluntaria de los mismos y el tratamiento anónimo de los datos. En un primer encuentro se trabajó con los alumnos avanzados y en el siguiente, con los ingresantes. En ambos casos (avanzados o ingresantes), se dividió azarosamente a la muestra en dos condiciones: quienes realizarían la actividad con información adicional y quienes no. A los sujetos que realizarían la tarea con información adicional en formato de gráfico de frecuencias, se los evaluó previamente con el objeto de determinar que contaban 
con una capacidad básica suficiente para la lectura de este tipo de sistemas, tal como se mencionó previamente. Los sujetos que no contaron con este requisito básico no fueron considerados para el estudio, debiéndose citar nuevos participantes hasta completar esa submuestra.

Luego, todos los participantes realizaron la tarea principal. Los participantes que realizaron la actividad sin información adicional trabajaron únicamente con el texto fuente que describía la situación sociocientífica. En cambio, quienes realizaron la actividad con información adicional trabajaron con el mismo texto fuente y cuatro gráficos de frecuencia vinculados al mismo.

\section{Análisis de datos}

Las producciones escritas de cada participante fueron analizadas en su totalidad constituyéndose en las unidades de análisis del estudio. Las categorías de análisis, que ya fueron descriptas con anterioridad (García-Milá et al., 2015; Eemeren et al., 2002), fueron las siguientes:

1. Texto argumentativo/no argumentativo:

a. Se consideraron textos argumentativos a aquellos que manifestaron uno o más puntos de vista con su respectiva defensa, compuesta esta de uno o más argumentos;

b. Por otro lado, un texto no argumentativo es aquel en el que hay ausencia de argumentos que sustenten un punto de vista.

Las categorías que se presentan a continuación (de la 2 a la 6) solo se aplican a los textos argumentativos.

2. Número de argumentos presentes en las composiciones argumentadas.

3. Diferencia de opinión principal:

a. Diferencia de opinión única no mixta;

b. Diferencia de opinión múltiple no mixta;

c. Diferencia de opinión única mixta; d. Diferencia de opinión múltiple mixta;

e. Diferencia de opinión no mixta con contextualización.

Es importante aclarar que la última categoría (diferencia de opinión no mixta con contextualización) se agregó a partir del trabajo de López y Padilla (2011). La misma permite considerar producciones que incluyen elementos del punto de vista contrario, pero no son incorporados a la argumentación.

4. Estructura argumentativa:

a. Argumentación simple;

b. Argumentación compleja: b1.) Múltiple, b2.) Coordinada, b3.) Subordinada y b4.) Combinada.

5. El sesgo confirmatorio (García-Milá et al., 2015) se utilizó para analizar solo las producciones escritas de los sujetos que tuvieron información adicional:

a. Se ignora;

b. Se lee incorrectamente (los datos implementados por los participantes no son los ofrecidos en el texto);

c. Se lee parcialmente (se utiliza solo una parte de la información brindada);

d. Se lee subjetivamente (a la información brindada los participantes le agregan una percepción personal del fenómeno);

e. Se lee correctamente (esta categoría fue agregada para identificar los casos en los que no se presenta sesgo confirmatorio).

6. Uso de información:

a. Argumento dado;

b. Argumento seminuevo;

c. Argumento nuevo.

Luego de realizar todas las categorizaciones previas, se continuó con el procesamiento cuantitativo 
de los datos. Este tuvo dos etapas claramente diferenciadas. En primer lugar, se realizó un análisis de correspondencias múltiples para observar simultáneamente las relaciones entre todas las variables estudiadas. En segundo lugar, se analizó cada una de las dimensiones argumentales propuestas en función de las dos variables independientes centrales del trabajo, es decir, momento de cursado y presencia del sistema representacional adicional. La influencia de ambas variables independientes fue considerada de manera independiente entre sí (efecto individual) y combinada (efecto de interacción). Vale recordar que las dimensiones categoriales analizadas fueron las siguientes:

- Producciones argumentativas y no argumentativas;

- Complejidad de las composiciones argumentativas: cantidad de argumentos en cada composición, estructura argumentativa, diferencia de opinión principal y sesgo confirmatorio;

- Uso de la información.

Esta segunda etapa analítica, de carácter confirmatorio, se basó predominantemente en análisis bivariados, aunque también se incluyó un análisis univariado de cada dimensión categorial como paso introductorio a su procesamiento. En casi todos los análisis bivariados, la significación estadística de las diferencias intergrupales se determinaron mediante pruebas no paramétricas (chi-cuadrado).

\section{Resultados}

\section{Análisis exploratorio inicial (correspondencias múltiples)}

En la Figura 1 se observa que en el factor 1 existe, por un lado, una oposición entre estudiantes ingresantes y la realización de textos no argumentativos; y por otro, estudiantes avanzados con un elevado número de argumentos, principalmente seminuevos y con estructuras argumentativas complejas. El factor 2 opone la argumentación simple, con respecto a la argumentación compleja. La Tabla 1 muestra una descripción detallada de los ejes factoriales.
Este análisis exploratorio multivariable permite un primer acercamiento a las características de la muestra, distinguiendo dos vinculaciones entre variables. Por un lado, se encuentra el estudiante avanzado con la capacidad de realizar textos argumentativos con una estructura argumentativa combinada, diferencia de opinión múltiple mixta y con uso del sistema informacional mediante la producción de argumentos con información dada; mientras que por otro lado, se encuentran los estudiantes ingresantes que se caracterizan por la exposición de una diferencia de opinión única mixta y no mixta y la argumentación simple.

\section{Producciones escritas argumentativas y no argumentativas}

En total se registraron 58 escritos argumentativos (80.6\%) y 14 escritos no argumentativos (19.4\%). En el segundo caso (escritos no argumentativos), los sujetos manifestaron solo su punto de vista, pero sin argumentarlo; o, directamente, no manifestaron punto de vista alguno. Una de las características principales de los escritos no argumentativos fue la escasez de coherencia. Por ejemplo, hubo textos que fueron producidos en base a una interpretación errónea de la consigna y desarrollando una argumentación sobre otra temática; o aquellos que sí explicitaban una postura adecuada en relación a la problemática, pero sus justificaciones carecían de sentido. También entran en este grupo textos cuya idea principal no pudo ser captada debido a la poca coherencia gramatical.

Por otro lado, se encontraron producciones descriptivas de la situación en las que no se adoptaba una postura, o no se la justificaba y se limitaba a repetir lo dicho en el texto fuente. Finalmente, se reportaron textos en los que se brindaban elementos prescriptivos o se exponían condiciones por las que se adoptaría un punto de vista ( «El Gobierno debería...», «Propongo la creación de...»).

Del total de producciones argumentativas, el $55.2 \%$ se manifestó a favor del cierre de la nuclear, el $37.9 \%$ estuvo en contra del cierre y un $6.9 \%$ dijo 


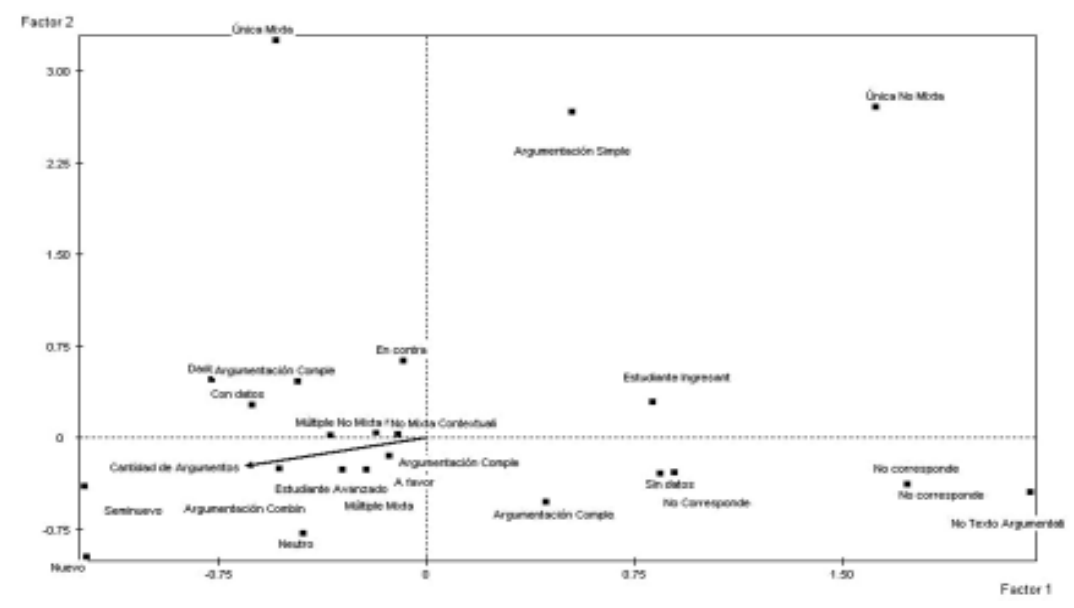

Figura 1. Representación gráfica de los ejes factoriales 1 y 2.

Tabla 1

Variables activas categóricas con mayor contribución en la conformación de los ejes factoriales 1 y 2

\begin{tabular}{|c|c|c|c|c|}
\hline & Variable & Categoría & V.T & $\mathrm{P}$ \\
\hline \multirow{13}{*}{ FACTOR 1} & Condición & Presencia del sistema informacional & -4.86 & 33.00 \\
\hline & Uso de información & Dado & -4.76 & 25.00 \\
\hline & Estructura argumentativa & Argumentación combinada & -3.67 & 29.00 \\
\hline & Texto argumentativo & Texto argumentativo & -3.11 & 58.00 \\
\hline & Momento de cursado & Estudiante avanzado & -2.92 & 41.00 \\
\hline & Diferencia de opinión & Múltiple mixta & -2.54 & 30.00 \\
\hline & ÁREA MEDIA & & & \\
\hline & Diferencia de opinión & No corresponde & 7.19 & 14.00 \\
\hline & estructura argumentativa & No corresponde & 7.19 & 14.00 \\
\hline & Posición & No corresponde & 7.19 & 14.00 \\
\hline & Condición & Ausencia del sistema informacional & 7.72 & 39.00 \\
\hline & Texto argumentativo & Texto no argumentativo & 9.03 & 14.00 \\
\hline & Uso de información & No corresponde & 9.19 & 43.00 \\
\hline \multirow{11}{*}{ FACTOR 2} & Diferencia de opinión & Múltiple mixta & -3.03 & 30.00 \\
\hline & Uso de información & No corresponde & -2.96 & 43.00 \\
\hline & Condición & Ausencia del sistema informacional & -2.71 & 39.00 \\
\hline & Momento de cursado & Estudiante avanzado & -2.50 & 41.00 \\
\hline & ÁREA MEDIA & & & \\
\hline & Año & Estudiante ingresante & 2.14 & 31.00 \\
\hline & Uso de información & Dado & 2.93 & 25.00 \\
\hline & Posición & En contra & 3.50 & 22.00 \\
\hline & Diferencia de opinión & Única mixta & 4.62 & 2.00 \\
\hline & Diferencia de opinión & Única no mixta & 4.75 & 3.00 \\
\hline & Estructura argumentativa & Argumentación simple & 6.77 & 6.00 \\
\hline
\end{tabular}

Nota: $\mathrm{V} . \mathrm{T}$ = valor del test; $\mathrm{P}=$ peso. 
tener una postura neutral. De las producciones no argumentativas, la mayoría perteneció al grupo de estudiantes iniciales (78.6\%), frente a un $21.4 \%$ de los estudiantes avanzados. En cuanto a los textos argumentativos, un $34.5 \%$ fue realizado por los estudiantes ingresantes, mientras que el $65.5 \%$ restante fue realizado por estudiantes avanzados. Estas diferencias fueron estadísticamente significativas $\left(\chi^{2}\right.$ $(1, n=72)=8.94, p<.05)$. En cambio, la presencia o no del sistema informacional, así como la combinación entre esta y el año de cursado, no mostraron efectos significativos en la producción de textos argumentativos y no argumentativos. De hecho, la mitad de los textos argumentativos fue realizada por los sujetos que tenían a su disposición el sistema informacional y la otra mitad, por los sujetos que no disponían de dicha información.

La mayor cantidad de textos no argumentativos fueron realizados por los sujetos ingresantes que no tenían información adicional (80\% de los textos no argumentativos). Este resultado estaría indicando que cuando no está presente un sistema de información adicional, los estudiantes novatos son los más perjudicados a la hora de escribir sus argumentos ante una determinada situación.

\section{Complejidad de las composiciones argumentativas}

Cantidad de argumentos en cada escrito argumentativo

La mayoría de los textos argumentativos registraron 3 o 4 argumentos (45.6\%). Siguieron aquellos con 2 (21.1\%) y 5 (17.5\%) argumentos, y, por último, solo un caso atípico con una frecuencia máxima de 8 . Cuando la cantidad de argumentos presentes en los textos argumentativos fue considerada en función del momento de cursado, se registraron valores promedios superiores en los estudiantes avanzados $(M=3.84$, $D E=1.48)$ en comparación con los ingresantes $(M=$ 2.25, $D E=1.07)$, con significatividad estadística $(t$ $(56)=-4.25, p<.001)$.
En cambio, la presencia del sistema informacional no arrojó diferencias significativas sobre la cantidad de argumentos, ya que los sujetos que dispusieron del mismo mostraron valores apenas superiores $(M=3.45$, $D E=1.82)$ a quienes no contaron con dicho recurso $(M=3.14, D E=1.22)$. Finalmente, tampoco se detectó un efecto interactivo significativo del momento de la carrera y de la presencia del sistema informacional sobre el volumen de argumentos. Aun así, en un sentido descriptivo, los estudiantes avanzados registraron una baja importante en la cantidad de argumentos al no contar con el sistema informacional (con información: $M=4.10, D E=.31$; sin información: $M=3.58, D E=$ .31); mientras que los ingresantes mostraron menores recuentos, independientemente de disponer $(M=2.20$, $D E=.43)$ o no $(M=2.30, D E=.43)$ de dicho recurso complementario.

\section{Estructura argumentativa}

La mitad de los escritos presentaron una estructura argumentativa compleja combinada (50.9\%). Le sigue en número la argumentación compleja múltiple, con un porcentaje del $21.1 \%$. Las estructuras argumentativas coordinadas y la simple única se presentaron en un $15.8 \%$ y en un $10.5 \%$, respectivamente. Finalmente, la estructura compleja subordinada se encontró en un solo caso.

Atendiendo a las distinciones en función del momento de la carrera, como puede observarse en la Tabla 2, los estudiantes ingresantes mayormente realizan una argumentación simple única y en menor porcentaje presentan argumentaciones complejas, mientras que los estudiantes avanzados se destacan por presentar estructuras argumentativas complejas, específicamente, combinadas.

En relación al número de argumentos y la estructura argumentativa, se halló que dentro de la argumentación compleja, aquellas producciones con un menor número de argumentos son de estructura múltiple o coordinada y aquellas con mayor cantidad de argumentos presentan una estructura combinada. La estructura puramente subordinada se presentó en 
Tabla 2

Estructura argumentativa en función del momento de la carrera

\begin{tabular}{lllrr}
\hline & & \multicolumn{2}{c}{ Momento de la carrera } & Total \\
\cline { 2 - 3 } & & Ingresante & Avanzado \\
\hline \multirow{2}{*}{$\begin{array}{l}\text { Estructura } \\
\text { argumentativa }\end{array}$} & Argumentación simple (única) & $4(66.7 \%)$ & $2(33.3 \%)$ & 6 \\
& Argumentación compleja múltiple & $5(38.5 \%)$ & $8(61.5 \%)$ & 13 \\
& Argumentación compleja coordinada & $5(55.6 \%)$ & $4(44.4 \%)$ & 9 \\
& Argumentación compleja subordinada & $1(100 \%)$ & 0 & 1 \\
& Argumentación compleja combinada & $5(17.2 \%)$ & $24(82.8 \%)$ & 29
\end{tabular}

Nota: $\chi^{2}(4, \mathrm{~N}=58)=10.327, p<.05$

un caso con dos argumentos. Los resultados indican un mayor uso de una argumentación combinada con 3 y 5 argumentos.

Específicamente el 31\% de estudiantes avanzados y el $10 \%$ de ingresantes utilizaron 2 argumentos múltiples y el $12 \%$ de estudiantes avanzados y el $6 \%$ de iniciales usaron 3 argumentos múltiples. El número de casos que utilizaron 2 argumentos coordinados es similar entre estudiantes ingresantes y avanzados. Finalmente, el número de estudiantes avanzados que utilizaron argumentos subordinados, tanto 1 como 2, sobrepasa en más del doble al de los estudiantes iniciales.

El análisis de la estructura argumentativa en función de la presencia o no del sistema informacional demostró que no existen diferencias estadísticamente significativas en relación a dicha variable, como puede observarse en la Tabla 3, evidenciándose una distribución equitativa en ambas condiciones $\left(\chi^{2}=4.71 ; \mathrm{gl}=4 ; p>.05\right)$.

Tabla 3

Estructura argumentativa en función de la presencia o no del sistema informacional

\begin{tabular}{lllrr}
\hline & & \multicolumn{2}{c}{ PSI } & \multicolumn{2}{c}{ Notal } \\
\cline { 3 - 4 } & & Sí & $3(50 \%)$ & 6 \\
Estructura & Argumentación simple (única) & $3(50 \%)$ & $7(53.8 \%)$ & 13 \\
argumentativa & Argumentación compleja múltiple & $6(46.2 \%)$ & $7(77.8 \%)$ & 9 \\
& Argumentación compleja coordinada & $2(22.2 \%)$ & 0 & 1 \\
& Argumentación compleja subordinada & $1(100 \%)$ & $12(41.4 \%)$ & 29 \\
\hline
\end{tabular}

Nota: PSI = Presencia del sistema informacional.

El análisis interactivo de las variables principales, estructura argumentativa en función del momento de la carrera y de la presencia o no del sistema informacional mostró una diferencia estadísticamente significativa para la condición ausencia del sistema informacional. En la Tabla 4 puede observarse que los estudiantes avanzados, a pesar de no tener información adicional, realizan escritos con argumentación compleja combinada, distinguiéndose de los estudiantes ingresantes que realizaron pocas argumentaciones complejas. 
Tabla 4

Estructura argumentativa en función del momento de la carrera y de la presencia o no del sistema informacional

\begin{tabular}{|c|c|c|c|c|}
\hline \multirow[b]{3}{*}{ Estructura argumentativa } & \multicolumn{4}{|c|}{ PSI } \\
\hline & \multicolumn{2}{|c|}{ Sí* } & \multicolumn{2}{|c|}{ No** } \\
\hline & Ingresante & Avanzado & Ingresante & Avanzado \\
\hline Argumentación simple (única) & $1(33.3 \%)$ & $2(66.7 \%)$ & $3(100 \%)$ & 0 \\
\hline Argumentación compleja múltiple & $3(50 \%)$ & $3(50 \%)$ & $2(28.6 \%)$ & $5(71.4 \%)$ \\
\hline Argumentación compleja coordinada & $1(50 \%)$ & $1(50 \%)$ & $4(57.1 \%)$ & $3(42.9 \%)$ \\
\hline Argumentación compleja subordinada & $1(100 \%)$ & 0 & 0 & 0 \\
\hline Argumentación compleja combinada & $4(23.5 \%)$ & $13(76.5 \%)$ & $1(8.3 \%)$ & $11(91.7 \%)$ \\
\hline
\end{tabular}

Nota: *Las diferencias no son significativas; ** $\left(\chi^{2}(3, \mathrm{~N}=29)=11.031, p<.05\right)$.

\section{Diferencia de opinión principal}

En relación a la diferencia de opinión, la mayor cantidad de escritos argumentativos (50.9\%) presentó una diferencia de opinión múltiple mixta; en segundo lugar, con un 31.6\%, una diferencia de opinión no mixta contextualizada; en menor proporción se encontraron las diferencias de opinión única no mixta (5.3\%); múltiple no mixta (8.8\%) y única mixta (3.5\%).

En cuanto a la distinción por el momento de la carrera, el número de estudiantes ingresantes que desarrollaron la diferencia de opinión múltiple mixta es del $24.1 \%$, mientras que el de estudiantes avanzados lo sobrepasa con el $75.9 \%$. También se encuentra la diferencia de opinión no mixta contextualizada en un $66.7 \%$ en contraste con un $33.3 \%$ de los estudiantes ingresantes. Respecto al resto de las diferencias de opinión, todas aparecen minoritariamente en la muestra con los resultados similares, no hallándose diferencias estadísticamente significativas.

En síntesis, el 34\% de las producciones presentó estructura argumentativa combinada y una diferencia de opinión múltiple mixta; el 12\% fue de estructura múltiple con diferencia de opinión múltiple mixta y un $10 \%$ fue de estructura combinada y una diferencia de opinión no mixta, pero con contextualización.

Tampoco se hallaron diferencias significativas en cuanto a la presencia del sistema de información con respecto a la diferencia de opinión y cuando se hizo intervenir todas las variables en juego (diferencia de opinión en función del momento de la carrera y de la presencia o no del sistema informacional).

\section{Sesgo confirmatorio}

El 51.7\% de la muestra que utilizó información adicional ignoró aquella que contradecía la propia tesis, mientras que el resto de los sujetos leyó la información de manera incorrecta (13.8\%), parcial (13.8\%), subjetiva (6.9\%) o correctamente (13.8\%).

La diferencia del sesgo confirmatorio en función del momento de la carrera no fue estadísticamente significativa, presentándose una distribución pareja del uso de la información en estudiantes ingresantes (se ignora $42.9 \%$ ) y avanzados (se ignora 57.1\%).

\section{Uso de información del sistema adicional (gráfico)}

Con respecto al uso de información, los resultados indican que el $86.2 \%$ de la muestra a la cual se le ofreció el sistema de información adicional construyó argumentos con la información dada en el gráfico, solo el 6.9\% generó un argumento seminuevo y el 6.9\% un argumento nuevo.

La distribución del uso de información en función del momento de la carrera no arrojó diferencias 
significativas, destacándose una distribución pareja entre ambas condiciones.

Indagando si la presencia de información adicional influyó en el número de argumentos desplegados, se observó que aquellas producciones que expusieron un mayor número de argumentos eran aquellas en las que los estudiantes contaban con la presencia del sistema informacional, pero la diferencia no fue significativa. Adicionalmente, se observó que los casos en los que se agregaron elementos seminuevos pertenecían a una argumentación de estructura combinada y aquellos donde se agregaron elementos nuevos pertenecían, uno a una argumentación de estructura múltiple y otro a una combinada.

En relación al número de argumentos, los casos en los que se agregó información seminueva presentaban entre 4 y 5 argumentos, mientras que uno de los que agrega información nueva presentó 4 argumentos y el otro, 8 .

Respecto de la diferencia de opinión, estos 4 casos que agregan información nueva o seminueva presentaron una diferencia de opinión múltiple mixta, todos ellos de estudiantes avanzados.

\section{Discusión}

Los resultados de esta investigación permiten enfatizar y concluir, al menos provisoriamente, varios aspectos de importancia. En primer lugar, la información empírica resultante del diseño aplicado podría contribuir tanto en el conocimiento de la competencia argumentativa como en la creación de material educativo para focalizar el trabajo en el aula universitaria.

Desde el primer punto de vista, y específicamente, nuestros datos podrían ayudar a evaluar el grado de idoneidad de las categorías de la teoría pragmadialéctica para el estudio de la argumentación escrita de estudiantes universitarios, a la vez de contribuir a la elaboración de un instrumento que pueda recoger estos datos en el futuro. Tiende a reportarse bajos niveles de complejidad argumentativa (López \& Padilla, 2011; Noemí \& Santibáñez, 2016), pero el problema parece ser que los criterios pragmadialécticos establecidos no son lo suficientemente realistas para establecer un tipo de normatividad. Este es un desafío para posteriores diseños metodológicos.

Las diferencias encontradas entre el estudiante avanzado y el estudiante principiante o ingresante (en Psicología) son claras en relación a varias dimensiones. En primer lugar, los estudiantes avanzados son capaces de (1) producir textos argumentativos con una estructura argumentativa combinada, (2) manifestar diferencia de opinión múltiple mixta y (3) ser capaces de integrar en la producción argumentativa información disponible. Por su parte, los estudiantes ingresantes muestran algunas tendencias altamente contrarias al estudiante avanzado, por ejemplo, (1) manifiestan diferencia de opinión única mixta y no mixta y (2) de argumentación simple.

Por otro lado, mientras que en los estudiantes avanzados la relación es menor cantidad de argumentos en función de menor cantidad de información; entre los estudiantes ingresantes, la baja cantidad de argumentos es independiente de la información disponible. Esto muestra claramente que en los estudiantes avanzados habría una conducta o hábito automático relativo al beneficio de integrar elementos del entorno en sus prácticas inferenciales. Desde el punto de vista teórico argumentativo, y probablemente en una relación casi directa al desarrollo cognitivo general, los estudiantes avanzados estén transitando desde marcos argumentativos (Hample, 2005) instrumentalistas y egocéntricos, a marcos argumentativos cooperativos y civiles donde importa alinearse con lo que dicen los demás y con la búsqueda de un argumento razonable.

Esta investigación también confirma una amplia literatura relacionada con la fuerza del sesgo de confirmación (ver resumen de esta literatura en Mercier \& Sperber, 2017), puesto que la mayoría de los participantes (sobre un 51.7\%) ignoró la información 
que contradecía el punto de vista adoptado, lo cual se suma a que un importante grupo de sujetos leyó la información de forma incorrecta (13.8\%), de forma parcial (13.8\%) o ya sea de forma subjetiva (6.9\%), y solo un pequeño porcentaje (13.8\%) la leyó correctamente. Esta tendencia sugiere, en el contexto de estudiantes universitarios argentinos que cursan la carrera de Psicología en distintos niveles, que debiera potenciarse decididamente actividades en el aula que tiendan a incorporar una división del trabajo cognitivo, al menos a nivel grupal para efectos de habituarse a la práctica de escuchar el punto de vista opuesto.

Otro hallazgo importante del estudio es que la combinación entre presencia o no del sistema informacional y el año de cursado no muestra efectos significativos en la producción de textos argumentativos y no argumentativos; en efecto, $\mathrm{y}$ como se sostuvo, la mitad de los textos argumentativos fue realizada por los sujetos que tenían a su disposición el sistema informacional y la otra mitad por los sujetos que no disponían de dicha información. Este hallazgo podría ser interpretado en línea con la prevalencia del sesgo de confirmación, en el sentido de que independientemente de los años en el sistema universitario, la información se utilizará en función de la preexistencia de un punto de vista adoptado.

En relación a las principales limitaciones del estudio, cabe mencionar la omisión del análisis del contenido semántico de las argumentaciones escritas. Aunque queda claro que el trabajo se enfocó desde el principio en la complejidad argumentativa (y sus componentes), la indagación de la narrativa argumental queda como un asunto pendiente a retomar en futuros trabajos.

En segundo lugar, el diseño no incluyó otras variables sociales o psicológicas que podrían influir en la toma de posición (por ejemplo, género, personalidad, prestigio académico, etc.). De igual modo que en el caso anterior, futuros estudios podrían considerar la interacción de estos factores con las variables explicativas aquí indagadas (momento de cursado y disponibilidad del sistema de representación externa).
Por último, también debe repararse en las dificultades registradas para lograr cantidades exactamente equivalentes de participantes entre las condiciones de estudio. Este tipo de problemática es habitual en nuestros estudios ya que, aunque se propone un abordaje experimental del fenómeno, el mismo tiene lugar en los contextos en los cuales los participantes se desenvuelven habitualmente (en este caso, la universidad). Por ese motivo, tal como se mencionó más arriba, se trató de un estudio principalmente cuasiexperimental, es decir, limitado por las restricciones planteadas por el propio terreno, pero con la intención de «naturalizar» la estrategia de indagación empírica.

Para finalizar, es importante considerar las implicancias educativas de estos hallazgos. Aunque, en general, se reconfirmaron niveles bajos de complejidad argumentativa escrita en estudiantes universitarios (en este caso de Psicología), esto se dio de manera más marcada al ingreso de la universidad, en comparación con los momentos más avanzados de cursado. Así, la planificación curricular en las asignaturas de nivel básico debería considerar este déficit argumental de base que el alumno trae desde su formación secundaria recientemente finalizada. En ese sentido, el docente debe enfrentarse a un doble desafío: por un lado, facilitar procesos de aprendizaje de la mayor calidad posible, a pesar de que el alumno cuente con un bajo desarrollo de las competencias argumentales; por otro lado, y de manera simultánea, promover el desarrollo de dichas competencias, lo cual puede darse, por ejemplo, incorporando esta cuestión como parte de los contenidos procedimentales y/o actitudinales del programa de estudio. Otra forma de incentivación de la argumentación, muy pertinente a las Ciencias Sociales (en general) y a la Psicología (en particular), podría basarse en la diversidad de puntos de vista conceptuales y metodológicos presentes e inherentes a estos campos de conocimiento. De esta manera, la formación no solo debería apuntar a un conocimiento conceptual profundo de cada modelo o perspectiva, sino también a la confrontación y revisión fundamentada entre las 
mismas. El mismo criterio aplica a la formación en las diversos abordajes metodológicos de la investigación en estas áreas (Leal-Carretero, 2017).

De concretarse exitosamente las acciones previas en los estudiantes ingresantes, sería esperable contar con mejores niveles de argumentación en los momentos más avanzados de la carrera. Aún así, en estas instancias, se agrega el elemento relativo a los sistemas de información adicionales para potenciar los procesos de argumentación. Por tanto, en este momento de la formación, también tendría sentido la incorporación de fuentes de información gráfica, adicionales del texto fuente de base.

\section{Conflicto de intereses}

Los autores declaran la ausencia de algún conflicto de interés (léase moral, económico, laboral o de investigación) para que el presente manuscrito sea sometido a evaluación.

\section{Responsabilidad ética}

Para la participación de los estudiantes universitarios en el presente estudio, se dejó expresa constancia del carácter voluntario de la misma y que el tratamiento de los datos se realizaría de manera anónima. Los participantes prestaron su consentimiento a ello.

Durante la totalidad del proceso de investigación se han respetado los lineamientos éticos requeridos en este tipo de investigaciones.

En el presente estudio no aparece dato alguno que permita la identificación de los participantes (completo anonimato).

\section{Referencias}

Arrosi, F., Axelrud, B., D’Agostino, M., \& Eisner, L. (2003). Competencias argumentativas en alumnos universitarios. In M. M. García (Ed.). Actas del Congreso Internacional La argumentación (pp. 656664). Buenos Aires: Universidad de Buenos Aires.
Bermejo-Luque, L. (2011). Giving Reasons: A Linguistic-Pragmatic Approach to Argumentation Theory. Netherlands: Springer.

Carrillo, M. C. (2010). Evaluación de textos argumentativos escritos en inglés de acuerdo con una propuesta integradora. In V. Castel \& L. Cubo de Severino (Eds.). La renovación de la palabra en el bicentenario de la Argentina. Los colores de la mirada lingüística (pp. 277-287). Mendoza: Editorial FFyL, UNCuyo.

Carrión, D., \& Castro, P. (2012). Tú, ¿¿Qué tipo de carne prefieres en tu hamburguesa? Una cuestión sociocientífica que promueve la argumentación. Memorias de la Maestría en Docencia de la Química, 2, 56-68.

Eemeren, F. van (2018). Argumentation Theory: A Pragma-Dialectical Perspective. Dordrecht: Springer.

Eemeren, F. van, \& Grootendorst, R. (2004). A Systematic Theory of Argumentation: The Pragma-Dialectical Approach. New York: Cambridge University Press.

Eemeren, F. van, Grootendorst, R., \& Snoeck-Henkemans, F. (2002). Argumentation: Analysis, Evaluation, Presentation. NJ: Lawrence Erlbaum Associates.

Errázuriz, M. C., \& Fuentes, L. (2010). Evaluación y desarrollo de una propuesta centrada en la producción de textos en estudiantes de primer año de Pedagogía en Educación General Básica. Sembrando ideas. Revista Educativa, 4, 38-53.

España, E., \& Prieto, T. (2010). Problemas sociocientíficos y enseñanza-aprendizaje de las ciencias. Investigación en la Escuela, 71, 17-24. Recuperado de http://investigacionenlaescuela.es/articulos/71/ R71_2.pdf

García-Milá, M., Pérez-Echeverría, M. P., Postigo, Y., Martí, E., Villarroel, C., \& Gabucio, F. (2015). ¿Centrales nucleares? ¿Si o no? ¡Gracias! El uso argumentativo de tablas y gráficas. Infancia y Aprendizaje, 39, 187-218. doi: 10.1080/02103 702. 2015.1111605

González, A., \& Puig, B. (2017). Analizar una problemática ambiental local para practicar la argumentación en clase de ciencias. Revista 
Electrónica de Enseñanza de las Ciencias, 16(2), 280-297. Recuperado de http://reec.uvigo.es/ volumenes/volumen16/REEC_16_2_6_ex1139.pdf

Hammer, L., \& Noemí, C. (2016). Relación entre pensamiento crítico y complejidad discursiva en estudiantes universitarios. In C. Santibáñez (comp.). Ecología argumentativa universitaria: de la realidad a los conceptos (pp. 187-208). Chile: Cosmigonon.

Hample, D. (2005). Arguing: Exchanging Reasons Face to Face. Mahwah, NJ: Erlbaum.

Hample, D., \& Irions, A. (2015). Arguing to Display Identity. Argumentation, 29, 389-416. doi: 10.1007/ s10503-015-9351-9

Jacobs, S. (2016). Employing and Exploiting the Presumptions of Communication in Argumentation: An Application of Normative Pragmatics. Informal Logic, 36(2), 159-191. doi: 10.22329/il.v36i2.4661

Johnson, R. (2000). Manifest Rationality: A Pragmatic Theory of Argument. Mahwah: Lawrence Erlbaum Associates. doi: 10.22329/il.v20i3.2282

Leal-Carretero, F. (2017). ¿Qué función cumple la argumentación en la metodología de la investigación en ciencias sociales? Espiral, Estudios sobre Estado y Sociedad, 24(70), 9-49.

Leitão, S. (2000). The potential of argument in knowledge building. Human Development, 43(6), 332-360. doi: 10.1159/000022695

López, E., \& Padilla, C. (2011). Grados de complejidad argumentativa en escritos de estudiantes universitarios de Humanidades. Praxis: Revista de Psicología, 20, 61-89.

Mercier, D., \& Sperber, H. (2017). The Enigma of Reason. A New Theory of Human Understanding. London: Allen Lane.
Noemí, C., \& Santibáñez, C. (2016). Para una pedagogía colectiva de la argumentación. In C. Santibáñez (comp.). Ecología argumentativa universitaria: de la realidad a los conceptos (pp. 251-260). Chile: Cosmigonon.

Popa, E. (2016). Criticism without Fundamental Principles. Informal Logic, 36(2), 192-216. doi: 10.22329/il.v36i2.4641

Roth, W., Pozzer-Ardenghi, L. , \& Han, J. Y. (2005). Critical Graphicacy. Understanding Visual Representation Practices in School Science. NL: Springer.

Santibáñez, C. (2014). ¿Para qué sirve argumentar? Problematizando teórica y empíricamente el valor y la función de la argumentación. Círculo de Lingüística Aplicada a la Comunicación, 58, 163205. doi: 10.5209/rev_CLAC.2014.v58.45474

Santibáñez, C. (2016). ¿Un hablante es argumentativamente complejo o robusto? Configurando criterios cualitativos desde la cognición cultural. In C. Santibáñez (comp.). Ecología argumentativa universitaria: de la realidad a los conceptos (pp. 223-250). Chile: Cosmigonon.

Siegel, H., \& Biro, J. (2008). Rationality, reasonableness, and critical rationalism: Problems with the pragmadialectical view. Argumentation, 22(2), 191-203. doi: 10.1007/s10503-007-9063-X

Smokrovic, N. (2015). Argumentation as a Means for Extending Knowledge. Croatian Journal of Philosophy, 15(44), 223-231.

Tindale, C. (2015). The Philosophy of Argument and Audience Reception. New York: Cambridge University Press. 


\section{María Agustina Tuzinkievicz}

Universidad Nacional de Rosario, Argentina.

Estudiante avanzada de Psicología en la Universidad Nacional de Rosario. Ayudante de Cátedra del curso Desarrollos Psicológicos Contemporáneos. En IRICE-CONICET-UNR (Argentina) investiga sobre argumentación en estudiantes universitarios.

\section{ORCID: 0000-0002-7443-9003}

matuzinkievicz@gmail.com

\section{Nadia Soledad Peralta}

Instituto Rosario de Investigaciones en Ciencias de la Educación, Argentina. Universidad Nacional de Rosario, Argentina.

Doctora en Psicología. Cuenta con publicaciones sobre conflicto sociocognitivo, aprendizaje colaborativo y argumentación en el ámbito universitario. Su línea de investigación refiere a la interacción sociocognitva y argumentación en contextos de aprendizaje académico.

\section{ORCID: 0000-0001-9950-6949}

Autor corresponsal: nperalta@irice-conicet.gov.ar; nadiasoledadperalta@gmail.com

\section{Mariano Castellaro}

Instituto Rosario de Investigaciones en Ciencias de la Educación, Argentina. Universidad Nacional de Rosario, Argentina.

Doctor en Psicología. Cuenta con investigaciones sobre interacción colaborativa infantil, aprendizaje colaborativo y educación. Su línea de investigación refiere a los procesos sociales y cognitivos implicados en el desarrollo cognitivo infantil y la construcción de conocimientos escolares.

ORCID: 0000-0001-5470-9662

castellaro@irice-conicet.gov.ar

\section{Cristián Santibáñez}

Universidad Católica de la Santísima de Concepción, Chile.

Sociólogo, Máster en Lingüística y Doctor en Lingüística. Desarrolla investigación en teoría de la argumentación, psicología del razonamiento, epistemología y cognición. Ha descrito la manera en que argumentan y razonan jóvenes, adultos y adultos mayores chilenos.

ORCID: 0000-0001-6755-3468

csantibanez@ucsc.cl 